\section{Analysis of ERK3 intracellular localization: dynamic distribution during mitosis and apoptosis}

F. Aredia, ${ }^{1,2}$ M. Malatesta, ${ }^{3}$ P. Veneroni, ${ }^{2}$

M.G. Bottone ${ }^{2}$

'Istituto di Genetica Molecolare CNR,

Pavia

2Dipartimento di Biologia e

Biotecnologie, Università degli Studi di

Pavia

${ }^{3}$ Dipartimento di Scienze Neurologiche,

Biomediche e del Movimento, Sezione di

Anatomia e Istologia, Università degli

Studi di Verona, Italy

\section{Abstract}

Extracellular signal-regulated kinases (ERK) 1, 2 and 3 are involved in cell proliferation and differentiation, and apoptosis; although ERK1/2 have been widely studied, limited knowledge on ERK3 is available. The present work aimed at investigating ERK3 distribution during cell cycle and apoptosis in human tumor HeLa cells. The analysis performed by double immunofluorescence and immunoelectron microscopy revealed that during interphase ERK3 is mainly resident in the nucleoplasm in association with ribonuclear proteins involved in early pre-mRNA splicing, it undergoes cell cycle-dependent redistribution and, during apoptosis, it remains in the nucleus in the form of massive nuclear aggregates, then moves to the cytoplasm and is finally extruded.

\section{Introduction}

Extracellular signal-regulated Ser/Thr kinases (ERKs), belonging to the MAPKs (mitogen-activated protein kinases) family, are evolutionarily conserved enzymes involved in several biological processes like proliferation, differentiation, inflammation and immune response. ${ }^{1}$ Upon activation by different kinases, they are translocated from the cytoplasm to the nucleus. ${ }^{2}$ The best characterized ERK1 and ERK2 enzymes share the highly conserved sequence Thr-Xxx-Tyr in the activation loop, which is the site of the activatory double phosphorylation. ${ }^{3}$ ERK3 and ERK4 belong to a distinct subfamily containing the motif Ser-Glu-Gly required for the stable interaction with (and consequent activation of) other kinases like MK5 (mitogen kinase 5). ${ }^{4}$
ERK3, first described in $1991,{ }^{5}$ was investigated with respect to its localization and properties, finding that it is differently expressed in human and rat. ${ }^{6,7}$ In fact, the C-terminal sequence of rat ERK3 does not contain the human KHLN motif ${ }^{5}$ that controls protein shuttling between ER (endoplasmic reticulum), ERGIC (ER-Golgi intermediate compartment) and Golgi apparatus. ${ }^{8}$ The cytoplasmic localization of ERK3 is regulated by a CRM1 (chromosome region maintenance 1 , also known as exoportin 1 (XP01) -dependent nuclear export mechanism, possibly through CRM1 ability to bind ERK3 in vitro. ${ }^{7}$ Bind et al. ${ }^{9}$ observed that the nuclear entry of ERK3 increases as cells progress through the $\mathrm{S}$ phase, while during the early phases of the subsequent cell cycle ERK3 localized back to the Golgi. In addition, they reported that the proteolytic cleavage of ERK3 is required for its release from the Golgi, given that the nuclear form of the protein was carboxy-terminally truncated..$^{9}$ This evidence supports the existence of a peculiar mechanism of ERK3 activation and suggests that ERK3 localization is strictly connected to its regulatory function of the cell cycle. In this respect, it has been reported that ERK3 overexpression triggers $\mathbf{G}_{1}$ arrest in different mammalian cell lines, ${ }^{7,10,1}$ and that ERK3 interacts directly with cyclin D3. ${ }^{12}$ Moreover, it is a substrate for other cell cycle effectors, such as the phosphatase Cdc14A, which is able to functionally interplay with ERK3 interactors (MK5 and cyclin D3), thereby linking regulation of both cell cycle progression and differentiation process, ${ }^{13}$ and cyclin B-Cdk1 that hyperphosphorylates ERK3 when cells enter mitosis and is dephosphorylated at the $\mathrm{M} / \mathrm{G}_{1}$ transition. ${ }^{14}$

The present work aims at depicting ERK3 distribution during interphase and mitosis in HeLa cells, paying particular attention to the ultrastructural analysis of ERK3 localization in interphase nuclear domains. Moreover, since a role of ERK1/2 in apoptosis activation has been reported, ${ }^{15}$ we investigated for the first time ERK3 localization in cells driven to apoptosis by drugs with a different mechanism of action, i.e. etoposide, a DNA topoisomerase II inhibitor that affects DNA replication, the RNA polymerase I inhibitor actinomycin $\mathrm{D}$, and paclitaxel that affects microtubule stability by binding to $\alpha$-tubulin. The pro-apoptotic experimental conditions we applied have been previously defined in our laboratory. ${ }^{16-18}$

\section{Materials and Methods}

\section{Cell cultures}

HeLa cells (from human uterine cervical cancer) were cultured in D-MEM supplemented with 10\% fetal bovine serum (FCS), $1 \%$ glu-
Correspondence: Dr. Maria Grazia Bottone, Dipartimento di Biologia e Biotecnologie "L. Spallanzani”, Università degli Studi di Pavia, Via Ferrata, 9, 27100 Pavia, Italy.

Tel. +39.0382 .986319 - Fax +39.0382 .986325$

E-mail: bottone@unipv.it

Key words: Apoptosis; ERK3; mitosis; immunoelectron microscopy; immunocytochemistry; snRNPs; splicing factor.

Contributions: FA, immunofluorescence experiments, analysis and discussion of the data; MM, electron microscopy experiments; PV, cell culture; MGB, method design, analysis and interpretation of results; FA, MM, MGB, manuscript writing. All authors approve the version to be published.

Conflict of interest: the authors declare no potential conflict of interest.

Acknowledgments: this research was supported by University of Pavia (FAR - Fondi di Ateneo per la Ricerca, 2014). We thank Dr. A.I. Scovassi (IGM-CNR, Pavia) for her skillful assistance during manuscript preparation. FA is a PhD student in Genetics, Cellular and Molecular Biology (University of Pavia, Italy). Confocal images were taken at the Centro Grandi Strumenti of the University of Pavia.

Received for publication: 25 September 2015 . Accepted for publication: 12 November 2015.

This work is licensed under a Creative Commons Attribution NonCommercial 3.0 License (CC BYNC 3.0).

(C) Copyright F. Aredia et al., 2015

Licensee PAGEPress, Italy

European Journal of Histochemistry 2015; 59:2571 doi:10.4081/ejh.2015.2571

tamine, $100 \mathrm{U} / \mathrm{mL}$ penicillin and $0.1 \mathrm{mg} / \mathrm{mL}$ streptomycin. Cells were grown as monolayer at $37^{\circ} \mathrm{C}$ in humidified atmosphere containing $5 \% \mathrm{CO}_{2}$. For immunofluorescence experiments, $1 \times 10^{5}$ cells were seeded on glass coverslips ( $22 \times 22 \mathrm{~mm}) ; 48 \mathrm{~h}$ later, the medium was replaced with fresh medium containing either $100 \mu \mathrm{M}$ etoposide (Sigma-Aldrich, Milan, Italy) or $1 \mu \mathrm{g} / \mathrm{mL}$ actinomycin D (Sigma-Aldrich) or $50 \mathrm{nM}$ paclitaxel (Sigma-Aldrich). After a 20 -h incubation, cells were washed with PBS (Phosphate Buffered Saline), fixed in 4\% formalin in PBS for 20 min at room temperature (r.t.), postfixed in $70 \%$ ethanol at $-20^{\circ} \mathrm{C}$ and kept for $24 \mathrm{~h}$ at $-20^{\circ} \mathrm{C}$.

\section{Double immunofluorescence}

For immunofluorescence, samples were rehydrated in PBS and incubated with the polyclonal antibody against ERK3 (Cell Signaling Technology, Euroclone, Milan, Italy, diluted 1:200) for $1 \mathrm{~h}$ at $37^{\circ} \mathrm{C}$ in a humidified chamber. 
For double immunofluorescence experiments, monoclonal antibodies to $\alpha$-tubulin (Molecular Probes, Invitrogen, Milan, Italy, diluted 1:50), Golgin-97 (Molecular Probes, diluted 1:50), Ki67 (Dako, Milan, Italy, diluted 1:20), (Sm)snRNP (small nuclear RNP, Y12) core protein (Abcam, Prodotti Gianni, Milan, Italy, diluted 1:250) and SC35 (Sigma-Aldrich, diluted 1:200), were applied for $1 \mathrm{~h}$. BrdU (5-bromo2'-deoxyuridine) incorporation was evaluated as previously described. ${ }^{19}$ Coverslips were washed in PBS and incubated with the appropriate Alexa-conjugated secondary antibodies (anti-rabbit Alexa 488 or anti-mouse Alexa 594, Molecular Probes) for $1 \mathrm{~h}$ at r. t. Finally, nuclei were counterstained Hoechst 33258 $(0.1 \mathrm{\mu g} / \mathrm{mL})$ for $15 \mathrm{~min}$ and coverslips were mounted with Mowiol (Calbiochem, Inalco, Milan, Italy).

\section{Fluorescence confocal microscopy}

For confocal laser scanning microscopy, we used a Leica TCS-SP system mounted on a Leica DMIRBE inverted microscope; for fluorescence excitation, an Ar UV laser at $364 \mathrm{~nm}$ was used for Hoechst 33258, Ar visible laser at $488 \mathrm{~nm}$ for Alexa 488 and He/Ne laser at 543 for Alexa 594. Spaced $(0.5 \mu \mathrm{m})$ optical sections were recorded using a $63 \mathrm{x}$ oil immersion objective. Images were collected in the $1024 \times 1024$ pixels format, stored on a magnetic mass memory and processed by the Leica Confocal Software.

\section{Immunoelectron microscopy}

For transmission electron microscopy, the cells were fixed with $4 \%$ paraformaldehyde in $0.1 \mathrm{M}$ phosphate buffer, $\mathrm{pH} 7.4$, at $4^{\circ} \mathrm{C}$ for $1 \mathrm{~h}$, washed with PBS, collected by scraping, then treated with $0.5 \mathrm{M} \mathrm{NH}_{4} \mathrm{Cl}$ in PBS for $45 \mathrm{~min}$ at $4^{\circ} \mathrm{C}$, dehydrated with ethanol and embedded in LR White resin. Ultrathin sections were placed on Formvar-carbon coated nickel grids and processed for immunocytochemistry. ${ }^{20}$ The anti-ERK3 antibody was used at the 1:10 dilution and revealed with a $12 \mathrm{~nm}$-gold-conjugated goat anti-rabbit secondary antibody (Jackson ImmunoResearch Laboratories Inc., LiStarFish, Milan, Italy). To reduce chromatin contrast and selectively reveal nuclear RNP constituents, the sections were treated with the EDTA regressive method ${ }^{21}$ and observed in a Philips Morgagni TEM equipped with a Megaview II camera.

\section{Results and Discussion}

We monitored the intracellular localization of ERK3 in HeLa cells by immunofluorescence experiments. During interphase, ERK3 was mainly detectable in the nucleoplasm, and only
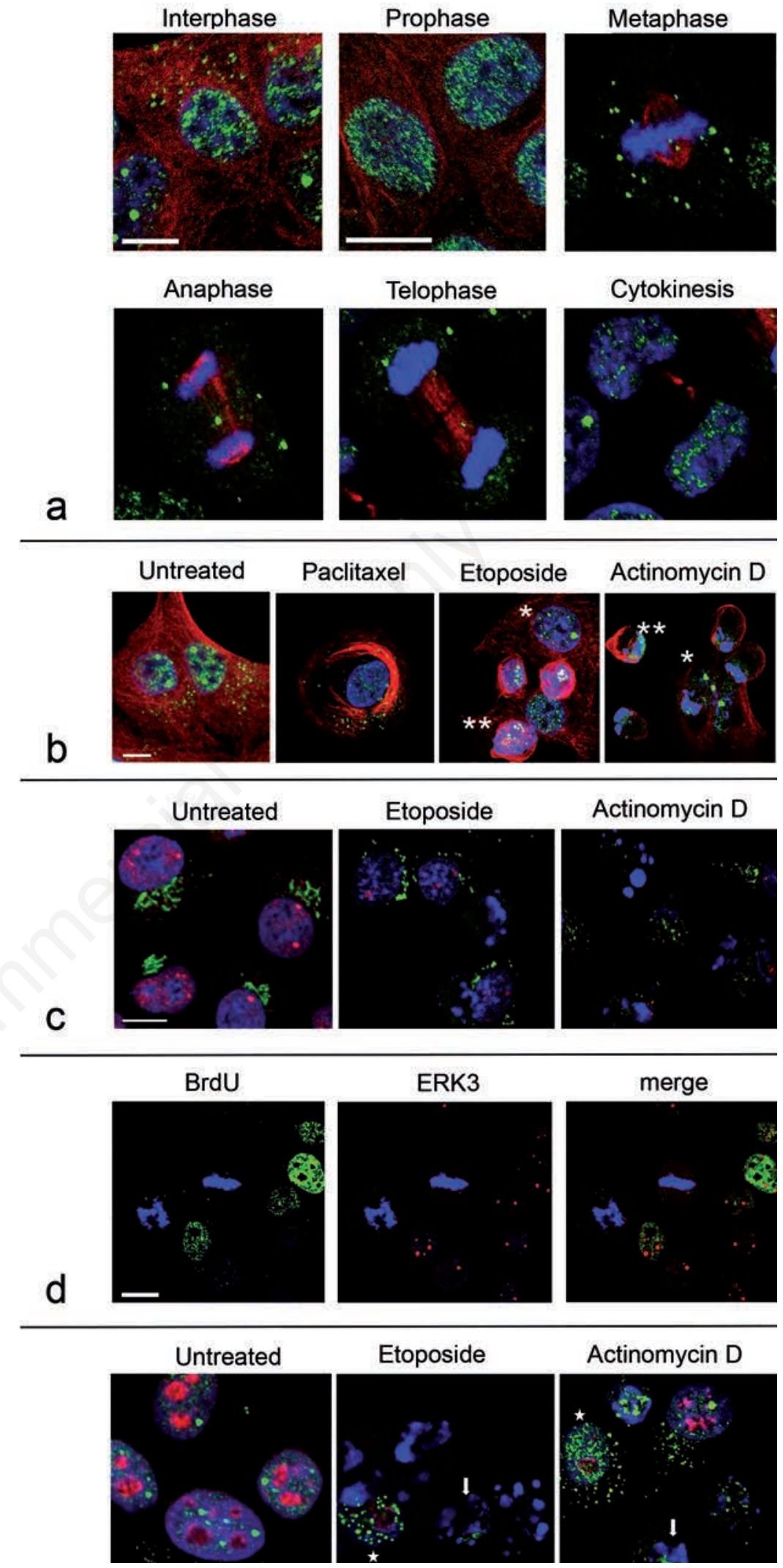

Actinomycin D

Figure 1. Confocal microscopy. a, b) Immunodetection of ERK3 (green fluorescence) and $\alpha$-tubulin (red fluorescence). c) Immunodetection of ERK3 (red fluorescence) and Golgin-97 (green fluorescence). d) Immunodetection of ERK3 (red fluorescence) and BrdU (green fluorescence). e) Immunodetection of ERK3 (green fluorescence) and Ki67 (red fluorescence); DNA is counterstained with Hoechst 33258 (blue fluorescence). ${ }^{*}$ Early apoptotic HeLa cells; ${ }^{* *}$ or arrow: late apoptotic HeLa cells. Scale bars: $20 \mu \mathrm{m}$. 
in some cells a cytoplasmic localization was observed, also in the vicinity of the perinuclear area (Figure 1a). The analysis of the intracellular distribution of ERK3 in the different phases of mitosis revealed that in interphase and prophase, it was scattered within the nucleus, whereas in metaphase it was distributed in homogeneous spots within the cell, where it remained until completion of chromosome segregation (during anaphase/ telophase); finally, after cytokinesis, ERK3 relocalized at the nuclear compartment (Figure 1a).

After the treatment with pro-apoptotic drugs (etoposide, actinomycin D or paclitaxel), in early apoptotic cells the ERK3 signal was visible as large nuclear spots, whereas during late apoptosis the signal was mainly diffused in the cytoplasm (Figure 1b). Later on, ERK3 was found to be extruded from the nucleus into the cytoplasm (Figure 1b). Bind et al. ${ }^{9}$ reported that a fraction of ERK3 localizes in the Golgi apparatus in a cell cycle-dependent manner: when the cells enter mitosis, ERK3 moves from the Golgi and returns back therein in the following cell cycle. However, in our experiments, the double staining for ERK3 and Golgin 97 revealed that, even if a fraction of ERK3 was distributed in the vicinity of this organelle, the two signals never overlapped (Figure 1c). This discrepancy could be due to the different antibodies used to detect the Golgi apparatus; in fact, the probe used by Bind et al. ${ }^{9}$ can also be applied for studying vesicular protein trafficking along the secretory pathway, whereas the probe used in the present study reacts with proteins located in the Golgi cisternae only. No correlation between ERK3 and Golgi apparatus was evident even in apoptotic cells, where the Golgi apparatus is morphologically and functionally rearranged (i.e., fragmentation, swelling and distension of the cisternae)..$^{22,23}$ In fact, during apoptosis ERK3 was detected in the cytoplasm, initially in the form of coarse aggregates, then in a more finely dispersed localization, but never colocating with the Golgi cisternae.

The distribution of ERK3 in relation to DNA replication foci was analyzed by immunofluorescence using BrdU incorporation, which reveals the newly synthesized DNA during the $\mathrm{S}$ phase. As evidenced in Figure 1d, the typical BrdU dots representative of the DNA synthesis sites never overlapped with the ERK3 immunolabeling, thus suggesting that this kinase does not colocate with the DNA replication sites.

The possible presence of ERK3 within nucleoli was investigated by a double immunolabeling with antibodies directed against the protein Ki67, which mostly accumulates in the nucleolus in interphase cells. ${ }^{24,25}$ Figure 1e shows that Ki67 was visible in untreated HeLa cells as distinct nuclear spots; when cells were submitted to the treatment with an apoptogenic drug (etoposide or actinomycin D), Ki67 remained associated to the nuclear compartment in early apoptotic cells, while in late apoptotic cells it was released as large aggregates within the cytoplasm. Remarkably, the nuclear signal for ERK3 never overlapped with the Ki67 staining, thus indicating that the two proteins do not share the same compartments.

The possible involvement of ERK3 in transcriptional and post-transcriptional processes was then investigated by both immunofluorescence and immunoelectron microscopy. By double immunofluorescence experiments, the distribution of ERK3 was evaluated in interphase cells in association with splicing factors known to localize at specific interchromatin domains involved in mRNA transcription and maturation: snRNPs (Y12), involved in early splicing at the transcriptional sites, ${ }^{26,27}$ and SC35, a non-RNP factor required for spliceosome assembly ${ }^{28}$ and mRNA transcription, 3' end processing and nucleus-to-cytoplasm export. $^{29,30} \mathrm{SC} 35$ is also considered as a reliable
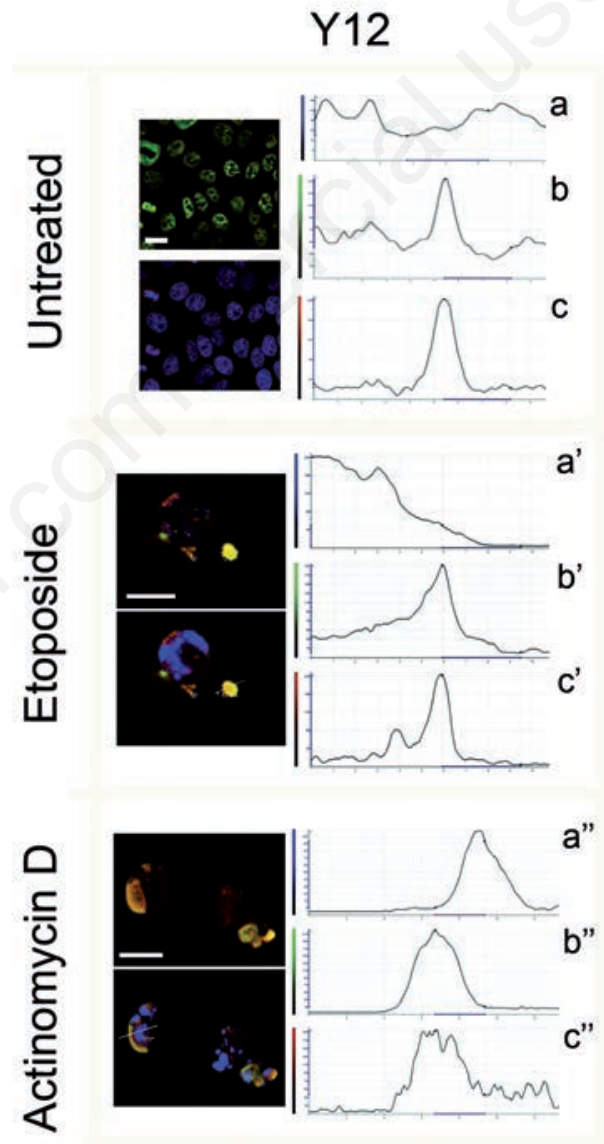

\section{SC35}
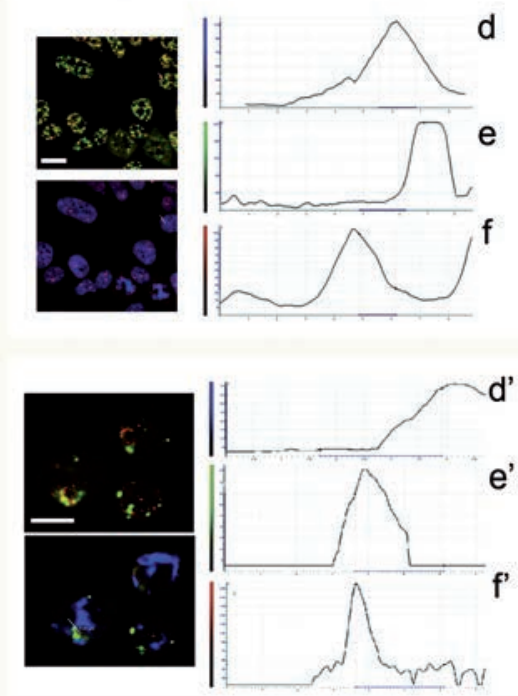

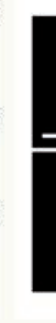

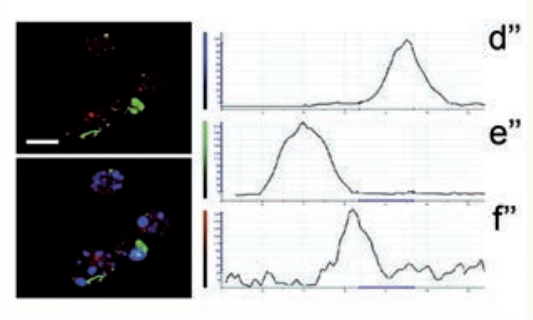

Figure 2. Confocal microscopy. Left: immunodetection of ERK3 (red fluorescence) and Y12 (green fluorescence). Right: immunodetection of ERK3 (red fluorescence) and SC35 (green fluorescence). DNA is counterstained with Hoechst 33258 (blue fluorescence). Each HeLa cell treatment condition is coupled with the respective graph of blue (a,a',a", d,d',d"), green (b, b',b",e, e', e') and red fluorescence (c,c',c', f, f', f') intensity for the colocalization analysis. Lines in the images represent the region where fusion of green/red fluorescence was measured. Scale bars: $20 \mp \mathrm{m}$. 

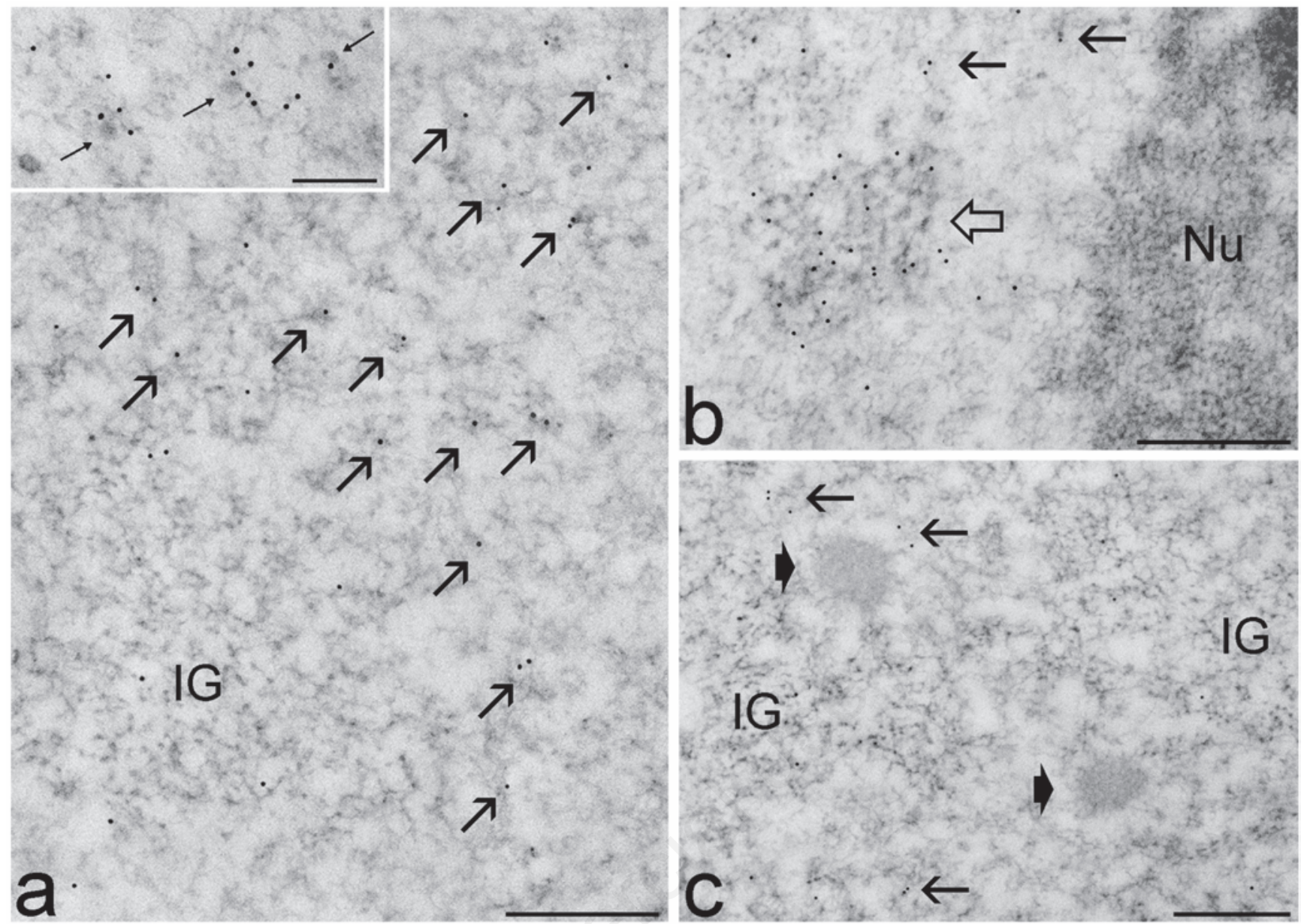

Figure 3. Transmission electron micrographs of HeLa cells, immunogold labelling with anti-ERK3 antibody. Most of labelling occurs on perichromatin fibrils (arrows), whereas interchromatin granules (IG) show only a weak signal. Perichromatin granules often show a specific labelling on the emerging RNP tails (small arrows, inset in a). Coiled bodies (open arrow) are always strongly labelled (b). No labelling has been found in nucleoli $(\mathrm{Nu}$ in $\mathrm{b}$ ) or in the interchromatin granule-associated zones (arrowheads in c). Scale bars: 500 $\mathrm{nm}$; inset bar: $250 \mathrm{~nm}$.

resenting the sites where transcription and cotranscriptional splicing of mRNAs occur), even when they were connected to perichromatin granules (vectors and storage site of already spliced pre-mRNA), ${ }^{27}$ but was absent from the interchromatin granules and from the interchromatin granule-associated zones, involved in the storage/assembly of U1snRNPs ${ }^{36}$ (Figure 3). ERK3 was also observed inside coiled (Cajal) bodies, i.e. the nuclear domains involved in multiple functions among which the intranuclear traffic of several splicing factors, including ( $\mathrm{Sm}) \mathrm{snRNPs} .{ }^{37}$ Consistent with the immunofluorescence results, ERK3 was never detected inside nucleoli. Taken together, these data strongly suggest that, in interphase cells, ERK3 plays a role in pre-mRNA transcription and/or splicing, maybe in association with (Sm)snRNPs, but it is not stored together with other splicing factors. Further experiments are required to define the functional role of ERK3 in these domains.

On the whole, our study on ERK3 in HeLa cells provides novel information about the intracellular location and dynamics of this kinase which has been attributed multiple roles in several cellular processes, such as spindle stability and metaphase-anaphase transition, ${ }^{38}$ dendrite morphogenesis,${ }^{39} \mathrm{~T}$ cell activation, ${ }^{40}$ endotelial cell functions, ${ }^{41}$ and tumorigenesis. ${ }^{42,43}$ We observed that during interphase, ERK3 is present mainly in the nucleoplasm, in association with RNP factors involved in early pre-mRNA splicing, and it undergoes extranuclear redistribution during mitosis, with no colocalization with Golgi cisternae. In addition, our study provides original data on the fate of ERK3 during apoptosis, when it initially remains in the nucleus in the form of massive nuclear aggregates, then moving to the cytoplasm and finally being extruded in association with other nuclear factors as it occurs for many other nuclear proteins that are involved in HERDS formation.

\section{References}

1. Cargnello M, Roux PP. Activation and function of the MAPKs and their substrates, the MAPK-activated protein kinases. Microbiol Mol Biol Rev 2011;75:50-83.

2. Coulombe P, Meloche S. Atypical mitogenactivated protein kinases: structure, regulation and functions. Biochim Biophys Acta 2007;1773:1376-87.

3. Bogoyevitch MA, Court NW. Counting on mitogen-activated protein kinases--ERKs 3, 4, 5, 6, 7 and 8. Cell Signal 2004;16:1345-54.

4. Déléris P, Rousseau J, Coulombe P, Rodier G, Tanguay PL, Meloche S. Activation loop phosphorylation of the atypical MAP kinases ERK3 and ERK4 is required for binding, activation and cytoplasmic relocalization of MK5. J Cell Physiol 2008;217:778-88.

5. Boulton TG, Nye SH, Robbins DJ, Ip NY, Radziejewska E, Morgenbesser SD, et al. ERKs: a family of protein-serine/threonine 
kinases that are activated and tyrosine phosphorylated in response to insulin and NGF. Cell 1991;65:663-75.

6. Cheng M, Boulton TG, Cobb MH. ERK3 is a constitutively nuclear protein kinase. J Biol Chem 1996;271:8951-8.

7. Julien C, Coulombe P, Meloche S. Nuclear export of ERK3 by a CRM1-dependent mechanism regulates its inhibitory action on cell cycle progression. J Biol Chem 2003;278:42615-24.

8. Teasdale RD, Jackson MR. Signal-mediated sorting of membrane proteins between the endoplasmic reticulum and the golgi apparatus. Annu Rev Cell Dev Biol 1996;12:27-54.

9. Bind E, Kleyner Y, Skowronska-Krawczyk D, Bien E, Dynlacht BD, Sanchez I.A Novel Mechanism for Mitogen-activated Protein Kinase Localization. Mol Biol Cell 2004;15: 4457-66.

10. Coulombe P, Rodier G, Pelletier S, Pellerin J, Meloche S. Rapid turnover of extracellular signal-regulated kinase 3 by the ubiquitinproteasome pathway defines a novel paradigm of mitogen-activated protein kinase regulation during cellular differentiation. Mol Cell Biol 2003;23:4542-58.

11. Crowe DL. Induction of p97MAPK expression regulates collagen mediated inhibition of proliferation and migration in human squamous cell carcinoma lines. Int $\mathrm{J}$ Oncol. 2004;24:1159-63.

12. Sun M, WeiY, Yao L, Xie J, Chen X, Wang H, et al. Identification of extracellular signalregulated kinase 3 as a new interaction partner of cyclin D3. Biochem Biophys Res Commun 2006;340:209-14.

13. Aaen Hansen C, Bartek J, Jensen S. A functional link between the human cell cycle-regulatory phosphatase Cdc14A and the atypical mitogen-activated kinase Erk3. Cell Cycle 2008;7:325-34.

14. Tanguay PL, Rodier G, Meloche S. C-terminal domain phosphorylation of ERK3 controlled by $\mathrm{Cdk} 1$ and $\mathrm{Cdc} 14$ regulates its stability in mitosis. Biochem J 2010; 428:103-11.

15. Cagnol S, Van Obberghen-Schilling E, Chambard JC Prolonged activation of ERK1,2 induces FADD-independent caspase 8 activation and cell death. Apoptosis 2006;11:337-346.

16. Rossi R, Montecucco A, Donzelli M, Denegri $\mathrm{M}$, Biamonti G, Scovassi A. DNA ligase I is dephosphorylated during the execution step of etoposide-induced apoptosis. Cell Death Differ 2002;9:89-90.

17. Fraschini A, Bottone MG, Scovassi AI, Denegri M, Risueño MC, Testillano PS, et al. Changes in extranucleolar transcription during actinomycin D-induced apoptosis. Histol Histopathol 2005;20:107-17.

18. Bottone MG, Soldani C, Tognon G, Gorrini C,
Lazzè MC, Brison 0, et al. Multiple effects of paclitaxel are modulated by a high c-myc amplification level. Exp Cell Res 2003;290:49-59.

19. Bottone MG, Soldani C, Veneroni P, Avella D, Pisu M, Bernocchi G. Cell proliferation, apoptosis and mitochondrial damage in rat B50 neuronal cells after cisplatin treatment. Cell Prolif 2008;41:506-20.

20. Costanzo M, Cisterna B, Vella A, Cestari T, Covi V, Tabaracci G, Malatesta M. Low ozone concentrations stimulate cytoskeletal organization, mitochondrial activity and nuclear transcription. Eur J Histochem 2015;59: 2515.

21. Bernhard W. A new staining procedure for electron microscopical cytology. J Ultrasruct Res 1969;27:250-255.

22. Jiang Z, Hu Z, Zeng L, Lu W, Zhang H, Li T, Xiao $\mathrm{H}$. The role of the Golgi apparatus in oxidative stress: Is this organelle less significant than mitochondria? Free Radic Biol Med 2011;50:907-17.

23. Bottone MG, Santin G, Aredia F, Bernocchi G, Pellicciari C, Scovassi AI. Morphological features of organelles during apoptosis: An overview. Cells 2013;2:294-305.

24. Bridger JM, Kill IR, Lichter P. Association of pKi-67 with satellite DNA of the human genome in early G1 cells. Chromosome Res 1998;6:13-24.

25. Endl E and Gerdes J. The Ki-67 protein: fascinating forms and an unknown function. Exp Cell Res 2000;257:231-7.

26. Lerner EA, Lerner MR, Janeway CA, Jr, Steitz JA. Monoclonal antibodies to nucleic acidcontaining cellular constituents: probes for molecular biology and autoimmune disease. Proc Natl Acad Sci USA 1981;78:2737-41.

27. Fakan S. The functional architecture of the nucleus as analysed by ultrastructural cytochemistry. Histochem Cell Biol 2004;122:83-93.

28. Fu XD, Maniatis T. Factor required for mammalian spliceosome assembly is localized to discrete regions in the nucleus. Nature 1990;343:437-41.

29. Lin R, Chen X, Li W, Han Y, Liu P, Pi R. Exposure to metal ions regulates mRNA levels of APP and BACE1 in PC12 cells: blockage by curcumin. Neurosci Lett 2008 8;440:344-7.

30. Bjork P, Jin S, Zhao J, Singh OP, Persson JO, Hellman U, Wieslander L. Specific combinations of SR proteins associate with single premessenger RNAs in vivo and contribute different functions. J Cell Biol 2009;184:555-568.

31. Puvion E, Puvion-Dutilleul F. Ultrastructure of the nucleus in relation to transcription and splicing: roles of perichromatin fibrils and interchromatin granules. Exp Cell Res 1996 15;229:217-25.

32. Bogolyubov D, Stepanova I, Parfenov V. Universal nuclear domains of somatic and germ cells: some lessons from oocyte interchromatin granule cluster and Cajal body structure and molecular composition. Bioessays 2009;31:400-9.

33. Biggiogera M, Pellicciari C. Heterogeneous ectopic RNP-derived structures (HERDS) are markers of transcriptional arrest. FASEB J 2000;14:828-34.

34. Biggiogera M, Bottone MG, Scovassi AI, Soldani C, Vecchio L, Pellicciari C. Rearrangement of nuclear ribonucleoprotein (RNP)-containing structures during apoptosis and transcriptional arrest. Biol Cell 2004;96:603-15.

35. Scovassi AI, Bottone MG, Biggiogera M, Pellicciari C. Dynamic relocation of nuclear proteins during the execution phase of apoptosis. Biochem Pharmacol 2008;76:1440-50.

36. Visa N, Puvion-Dutilleul F, Bachellerie JP, Puvion E. Intranuclear distribution of U1 and U2 snRNAs visualized by high resolution in situ hybridization: revelation of a novel compartment containing U1 but not U2 snRNA in HeLa cells. Eur J Cell Biol 1993;60:308-21.

37. Hebert MD. Signals controlling Cajal body assembly and function. Int $\mathrm{J}$ Biochem Cell Biol 2013;45:1314-7.

38. Li S, Ou XH, Wang ZB, Xiong B, Tong JS, Wei $\mathrm{L}$, et al. ERK3 is required for metaphaseanaphase transition in mouse oocyte meiosis. PLoS One. 2010;5 pii: e13074.

39. Brand F, Schumacher S, Kant S, Menon MB, Simon R, Turgeon B, et al. The extracellular signal-regulated kinase 3 (mitogen-activated protein kinase 6 [MAPK6])-MAPK-activated protein kinase 5 signaling complex regulates septin function and dendrite morphology. Mol Cell Biol 2012;32:2467-78.

40. Marquis M, Boulet S, Mathien S, Rousseau J, Thébault P, Daudelin JF, et al. The nonclassical MAP kinase ERK3 controls T cell activation. PLoS One 2014;9:e86681.

41. Wang W, Bian K, Vallabhaneni S, Zhang B, Wu RC, O'Malley BW, et al. ERK3 promotes endothelial cell functions by upregulating SRC-3/SP1-mediated VEGFR2 expression. J Cell Physiol 2014;229:1529-37.

42. Long W, Foulds CE, Qin J, Liu J, Ding C, Lonard DM, et al. ERK3 signals through SRC-3 coactivator to promote human lung cancer cell invasion. J Clin Invest 2012;122:1869-80.

43. Radu M, Semenova G, Kosoff R, Chernoff J. PAK signalling during the development and progression of cancer. Nat Rev Cancer 2014;14:13-25. 